\title{
SynRGen: An Extensible File Reference Generator
}

\author{
Maria R. Ebling and M. Satyanarayanan \\ School of Computer Science \\ Carnegie Mellon University \\ $\{$ mre,satya $\} @$ cs.cmu.edu
}

\begin{abstract}
SynRGen, a synthetic file reference generator operating at the system call level, is capable of modeling a wide variety of usage environments. It achieves realism through trace-inspured micromodels and flexubulity by combining these micromodels stochastically. A micromodel is a parameterized prece of code that captures the distinctive signature of an application. We have used SynRGen extensively for stress testing the Coda File System We have also performed a controlled experiment that demonstrates SynRGen's ablity to closely emulate real users - withun $20 \%$ of many key system variables In this paper we present the rationale, detaled design, and evaluation of SynRGen, and mention ts applicability to broader uses such as performance evaluation
\end{abstract}

\section{Introduction}

Transforming a file system from an initial prototype into a fully deployed system is a process fraught with hazard. Many insidious bugs will only be triggered under heavy loads and extended usage. But fear of serious failures, involving loss of data and lengthy downtime,

Sources for SynRGen are avalable vá AFS (/afs/cs cmu edu/project/coda-synrgen)or via anonymousFTP from ftp cs cmu edu (project/synrgen) For questions or comments related to SynRGen, please send e-mail to synrgen@cs cmu edu

This work has been supported by the Advanced Research Projects Agency (Avionics Lab, Wright Research and Development Center, Aeronautical Systems Division (AFSC), U S Air Force. Wright-Patterson AFB, Ohı, 45433-6543 under Contract F33615-90-C-1 465, ARPA Order No 7597 and Hanscom AFB. Contract F19628-93C-0193. ARPA Order No A700), the National Science Foundation (Grant No ECD 8907068), the IBM Corporation, Digital Equipment Corporation, and Xerox Corporation The views and conclusion expressed in this paper are those of the authors, and should not be interpreted as those of the funding organizations or Carnegie Mellon University

Permission to copy without fee all or part of this material is granted provided that the copies are not made or distributed for direct commercial advantage, the ACM copyright notice and the title of the publication and its date appear, and notice is given that copying is by permission of the Association of Computing Machinery. To copy otherwise, or to republish, requires a fee and/or specific permission.

SIGMETRICS 94- 5/94 Santa Clara, CA. USA

(C) 1994 ACM 0-89791-659-x/94/0005..\$3.50 deters many potential users. How, then, can implementors hope to increase the robustness of their system?

SynRGen is our solution to this problem. Configuration files, describing the behavior of real users and the characteristics of their data, are used by SynRGen to construct programs called synthetic users. When executed, a synthetic user generates references emulating the modeled users. By stress testing with a wide variety of synthetic users, an experimental system can be brought to an acceptable level of robustness.

SynRGen's usefulness extends well beyond stress testing. Since synthetic users can be parameterized and since the generated workload is reproducible, they can be used as the basis of a family of benchmarks for performance evaluation Further, the logistical and privacy problems inherent in exporting reference traces can be avoided by exporting a synthetic user representative of those traces. Most importantly, SynRGen allows a system to be subjected to hypothetical or anticipated usage scenarios. For example, one may wish to study the behavior of a file system when the extent of writesharing, the degree of locality or the distribution of file sizes differs substantially from that of any currently available environment.

We have used SynRGen extensively in the development of the Coda File System[16]. Our expenence confirms that it is an invaluable tool for file system development. We have also shown that the synthetic users produced by SynRGen can closely emulate the impact of real users on system resources. Specifically, our experiments indicate that SynRGen can emulate a group of users in an edit/debug cycle within $20 \%$ of key system variables such as network load and server CPU usage

Our description of SynRGen begins with a discussion of the primary factors that influenced its design. We then describe its architecture and implementation. The results of a controlled experiment exploring SynRGen's ability to emulate real users are presented in Section 4. We conclude the paper with a discussion of potential refinements and a survey of related work. 


\section{Design Rationale}

\subsection{Combining Realism with Flexibility}

The dominant desıgn consideration for SynRGen was our need to characterize a wide variety of usage environments, including aspects such as the physical characteristics of files, the behavior of users and programs, and the scale of the system. In building SynRGen, we had to carefully balance the degree of realism achieved with the amount of flexibility possible.

Realism can be viewed as the measure of correspondence along a number of dimensions[6]. The dimensions of interest may vary considerably. For example, in one experiment the only variable of interest may be server CPU utilization. In contrast, a more detailed experiment might include many system variables such as cache hit ratio at clients, volume of client-server traffic, and disk traffic at the server. The ultimate degree of realism is to replay an actual file reference trace. Unfortunately, traces can be extrapolated only in limited ways. Parameters such as file sizes and interarrival times can be scaled with relative ease. But there is no mechanical way to modify more complex aspects such as the degree of write-sharing between users.

SynRGen achieves realism through trace-inspired micromodels. File reference traces of applications reveal distinctive patterns or signatures in their file access behavior. A micromodel captures the signature of a particular application in a parameterized function. As an example, consider a hypothetical $C$ compiler that opens and reads a ".c" file, opens and reads a number of ".h" files, creates an empty ".o" file, writes to that file, and finally closes it. The specific ".c" file referenced, the number and identity of ".h" files, the sizes of each file. and many other details vary from execution to execution. Yet an examination of traces from many such executions will reveal the general pattern described above. A micromodel for this hypothetical $\mathrm{C}$ compiler would capture this distinctive signature, parameterizing the details of interest that vary between executions.

SynRGen achieves flexibility because experimenters can stochastically combine micromodels to capture workloads representative of a particular class of users. They can also specify parameter values at runtime and during configuration. Rather than wiring in the degree of realism, our approach defers this decision to the experimenter.

We expect most experimenters will begin by using existing micromodels, simply setting parameter values appropriately. If they find that no micromodel exists for an important application or if they find that the existing micromodel is not sufficiently accurate. they will either create a new micromodel or improve the existing one. An important aspect of our approach is that it is possible to substantially separate the efforts of the modeler and experimentermicromodels encapsulate the work of the former.

\subsection{File System Independence}

A goal in developing SynRGen was to compare the performance of alternative implementations of a particular file system API (application program interface) for identical user communities. For example, one might want to compare AFS[14], NFS[12], Sprite[11] and Coda[16]. This requires that the reference stream generated by SynRGen had to be at the level of abstraction common to these file systems, in this case the Unix file system API.

References generated at the file system API means that semantic constraints at that level must be respected. For example, in the Unix API, one cannot delete a directory unless it is empty; nor can one read from a file until it has been opened. Rather than trying to capture these API-specific constraints in some declarative form (such as a table), we chose to embed them in micromodels written in arbitrary $\mathrm{C}$ code. It is then the responsibility of the micromodel's author to ensure that API-specific constraints are met. Further, the mechanism for stochastically combining micromodels is APl-independent because the micromodels encapsulate all knowledge of the API.

A consequence of this decision is that SynRGen is not restricted to generating file system references. By writıng appropriate micromodels, SynRGen could equally well generate, for example, synthetic SQL database queries or disk I/O references. Although our experience with SynRGen has been limited to file reference generation. we do not foresee any obstacles to its broader use.

\subsection{Parameterizing File Locality}

The performance of virtually every file system is critically dependent upon design assumptions regarding the degree and nature of locality of file reference. If these assumptions do not hold in a particular environment, the performance of the file system could be significantly different from expectations. We wanted the ability to study the effect of changing the locality of reference substantially.

In order to conduct such a study without recoding every micromodel, one needs the ability to convey interfile locality information between independently-authored micromodels. For example, if a user examines the attributes of a given file, he or she is likely to look at the contents of that file next. Somehow, we must capture this temporal locality of reference. We use pathname iterators to meet this requirement. A pathname iterator is simply a procedure that encapsulates locality information. Each call to a pathname iterator yields the name of a file or directory; the stream of names generated by successive calls exhibits the interfile locality modeled by that iterator. To use SynRGen micromodels with different locality patterns, one merely invokes them with different pathname iterators.

In principle, a similar mechanism could provide a choice of intrafile locality models. However, intrafile locality tends to be 


\begin{tabular}{|c|c|c|c|c|c|}
\hline \multirow[b]{2}{*}{ Physical Characteristic } & \multicolumn{5}{|c|}{ Volume Type } \\
\hline & User & Project & System & BBoard & All \\
\hline Total Number of Volumes & 786 & 121 & 72 & 71 & 1050 \\
\hline Total Number of Directories & 13955 & 33642 & 9150 & 2286 & 59033 \\
\hline Total Number of Files & 152111 & 313890 & 113029 & 144525 & 723555 \\
\hline Total Size of File Data (MB) & 1700 & 7000 & 1500 & 560 & 11000 \\
\hline Absolute Depth & $4.3 \quad$ (1 3$)$ & $6.3 \quad(21)$ & $60 \quad(13)$ & 53 & $5.7 \quad(20)$ \\
\hline Relative Depth & $3.3 \quad$ (1 3$)$ & $5.2 \quad(20)$ & $4.0 \quad(12)$ & $(08)$ & $4.5 \quad$ (19) \\
\hline File Size (KB) & $10.3(650)$ & $24.0(1457)$ & $16.4(72.6)$ & $2.6 \quad(7.0)$ & $19.1(1180)$ \\
\hline Directories/Directory & $3.6(134)$ & $3.0 \quad(45)$ & $3.6(104)$ & $6.8 \quad(194)$ & $3.2 \quad(83)$ \\
\hline Files/Directory & $14.6(306)$ & $16.2 \quad(356)$ & $15.9(369)$ & $66.9(1424)$ & 15.7 \\
\hline Hard Links/Directory & $3.7 \quad(124)$ & (15) & $4.0 \quad$ (39) & $0.0 \quad(00)$ & $(57)$ \\
\hline Symbolic Links/Directory & $4.1 \quad(10.1)$ & (7.5) & $13.6 \quad(453)$ & $6.0 \quad(259)$ & (249) \\
\hline
\end{tabular}

This table summarızes varıous physical characterıstics of system, user, project, and bulletin board ("bboard") volumes in AFS at Carnegie Mellon University in early 1990 These data were obtained via static analysis. We present only the mean and standard deviation (1n parenthesis) here. The full data are represented in cumulative distribution functions Absolute depth is measured from "/afs/cs cmu edu/user" for user volumes, "dfs/cs cmu edu/project" for project volumes, and so on Relatıve depth is measured from the volume root

Table 1 Sample Physical Characteristics by Volume Type

specific to an application rather than being a function of the usage environment. Hence we expect SynRGen's micromodels to capture intrafile locality internally. For instance, a micromodel for the more program would open a file and read all or the initial part of the file sequentially, while a micromodel for a database or a linker/loader would open a file and access portions of the file randomly

\section{Architecture and Implementation}

The design rationale presented in the previous section leads directly to the architecture of SynRGen. The core of SynRGen consists of a set of preprocessors that transform configuration files into executable code. linking in the specified micromodels from a library. Synthetic user executables are generated for each type of user specified by the configuration files. Running a synthetic user results in references corresponding to that type of user.

An experiment consists of subjecting a candidate system to a collection of synthetic users. To emulate a timesharing environment, multiple synthetic users are run on the same machine. When emulating a distributed workstation environment, each synthetic user is run on a different client machine. The system-specific instrumentation necessary for monitoring the impact of synthetic users on clients, servers. and the network must be provided externally.

In the following sections, we describe SynRGen at the next level of detall. We first present the abstractions supported by SynRGen, and then discuss how each abstraction is specified. We complete the section by giving the status of our implementation.

\subsection{SynRGen Abstractions}

Our design is based upon two key abstractions: volumes and user classes. The volume abstraction provides the basis for modeling the layout and storage characteristics of the file system, while the user class abstraction provides the basis for modeling user activity.

A volume is a subtree of files and directories exhibiting a unique combination of physical characteristics. Each characteristic, such as file size and directory fan-out, is described by a distinct volumespecific stochastic distribution. For simplicity, we assume volumes are mounted only at the root of the file system hierarchy. Table 1 summarizes the characteristics observed in different types of volumes from a representative file system

A user class corresponds to a stochastic finite state machine. States in the FSM represent distinct user behaviors, while transitions represent a user changing from one behavior to another. For example, a behavior might be "programming" or "document processing". Each behavior consists of some set of possibly repetitive actions, corresponding to subtasks performed within this behavior. The actions associated with a "programming" behavior might include "searching header files", "editing" and "compiling", while the actions associated with a "document processing" behavior might include "editing" and "formatting" Actions exhibit distinctive file access characteristics, and correspond to micromodels 


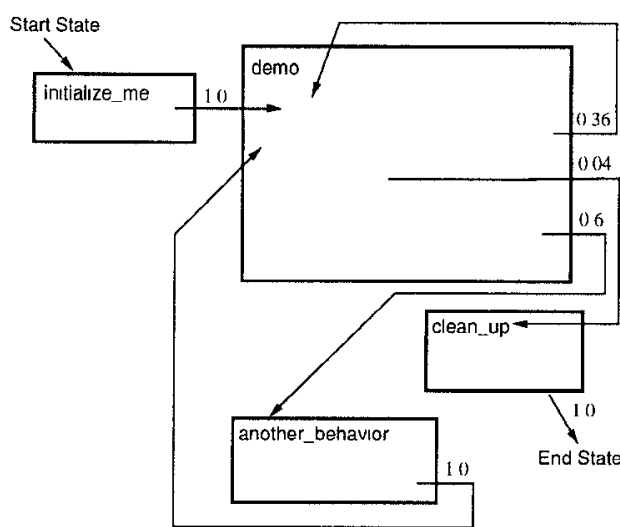

(a) Complete Finite State Machine

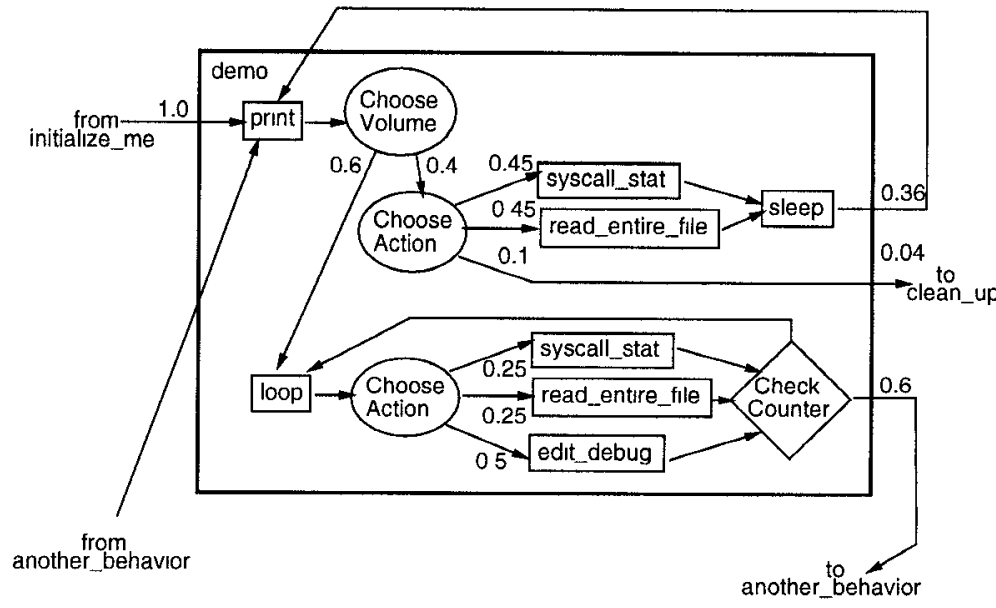

(b) Detail of demo Behavior

Flgure (a) shows the finite state machine for a hypothetical user class Each of the states initialize_me, demo, clean-up, and another_behavior represent user behaviors Figure (b) shows the detalled contents of one of these behaviors, demo Each small rectangle in this figure, such as edit_debug and read entire. $f i l e$, corresponds to an action, these actions are implemented in micromodels In both figures, arcs represent transitions between user behaviors, and the numbers on the arcs indicate transition probabilities

\section{Figure 1: User Class Finite State Machine}

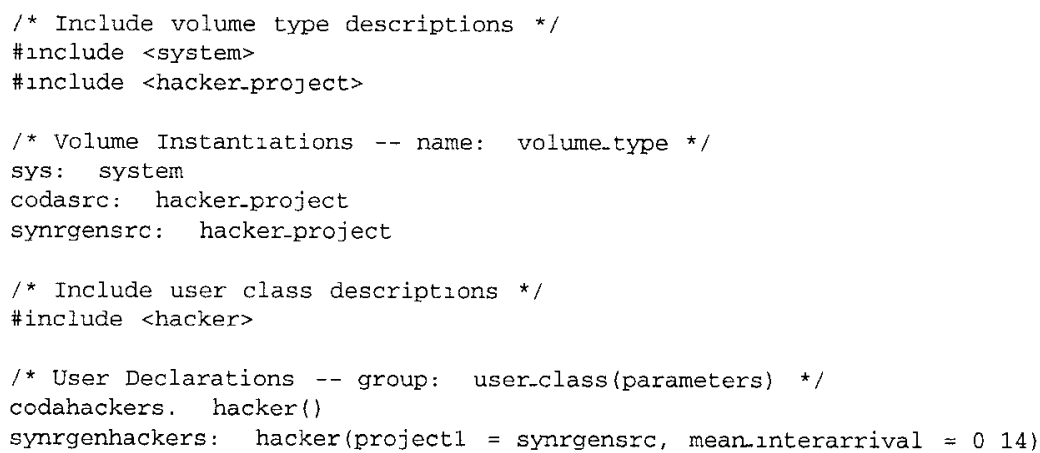

Figure 2: Sample System Configuration File

This figure shows a sample system configuration file Notıce that the synrgenhackers user declaration redefines the default value of the \$project 1 variable to "synrgensre" and the value of the meaninterarrival variable to 014

\begin{tabular}{llllll}
\multicolumn{2}{l}{ FILESIZE: } & \multicolumn{2}{l}{ DIRS per DIRECTORY. } \\
007 & 0 & 0.67 & 0 & \multicolumn{2}{l}{ SYMLINKS per DIRECTORY: } \\
010 & 100 & 081 & 1 & 091 & 0 \\
013 & 200 & 089 & 2 & 0.95 & 1 \\
$\ldots$ & & 092 & 3 & 0.98 & 3 \\
$\cdots$ & & 095 & 4 & 099 & 9 \\
099 & 400000 & &. & 100 & 90 \\
100 & 10000000 & 100 & 100 & &
\end{tabular}

This figure, containing excerpts of the project volume configuration file, shows three physical characteristics of this type of volume This file contains the inverse transformation of the CDF for each characteristic For example, $13 \%$ of all files in project volumes contann no more than 200 bytes of data The data detals the summary presented in Table 1

Figure 3: A Portion of a Volume Configuration File 
Figure 1(a) shows an example of a user class. This class of users exhibits four behaviors initialize_me, demo. another_behavior and clean_up. The arcs in this figure represent transitions between behaviors; the numbers represent the probability of taking a particular transition Figure 1(b) details a sungle behavior (demo) of that user class The actions associated with the demo behavior include syscall_stat, readentire_file and edit_debug.

Our representation of a user class closely resembles a user behavior graph, as defined by Ferrari[6] Each behavior in a SynRGen user class corresponds to a node in the behavior graph, and each transition to an arc.

\subsection{Configuration Files}

SynRGen's volume and user class abstractions are described in configuration files which are transformed by preprocessors. The mkclass preprocessor transforms a user class into a $\mathrm{C}$ program representing a synthetic user. The mkvol preprocessor transforms a volume description into a $\mathrm{C}$ data structure accessed by micromodels through library routines. To sımplify the compilation and linking of synthetic users, we provide a third preprocessor. mksynrgen. that takes a system configuration file and generates shell scripts.

We describe SynRGen further with a set of examples Figure 2 shows a typical system configuration file. The first section of this file defines volume descriptions by including volume configuration files, system.vol and hacker-project.vol In the next section. we instantiate three volumes: sys, codasrc, synrgensrc. The first volume is of type system, and the other two are of type hacker-project. The syntax resembles the way in which $C$ programs include header files to obtain typedef definitions and then instantiate variables of those types. In a similar manner, the rest of the file obtains definitions of the user class hacker and instantiates two different instances of this type of user. The instances differ in that codahackers uses the default set of parameters, while synrgenhackers redefines certain parameters.

Figure 3 depicts a portion of a volume configuration file. This file can contain up to six sections, each describing a physical characteristic of the file system hierarchy. The physical characteristics are: the file size; the number of files, symbolic links, hard links and directories per directory; and the relative depth. The characteristics are described using the inverse transformation of the corresponding CDF (cumulative distribution function). This information is transformed into a data structure used by the volume information routines (e.g. get_Filesize) accessible to micromodels.

Figure 4 depicts the user class configuration file corresponding to Figure 1. The heart of the user class definition consists of the description of individual behaviors such as initialize_me, demo, another.behavior and clean-up. Descriptions of be- haviors can use either arbitrary $\mathrm{C}$ code or our syntactic constructs for commonly encountered control flows. The demo behavior. for example, uses our syntactic constructs. After entering the demo behavior, the program will stochastically choose a volume in which to operate (either the \$project1 or the sys volume). If the project volume is chosen, the program will loop, stochastically choosing one of three actions (syscall_stat, readentire-file or edit_debug) on each iteration These actions are micromodels. If the sys volume is chosen instead, the program will stochastically choose to perform elther a syscall_stat, a readentire-file or a transition to the clean-up behavior. Notice that each of the micromodels take a pathname iterator, Fractile_Falloff(), as a parameter.

Parameters to the user class can be accessed within the configuration file by prepending a $\$$ to their name, e g. \$project 1 . These variables are bound to a default value when they are declared, but can be rebound either at configuration time or at run time. Run time binding takes precedence over configuratıon time binding, which in turn takes precedence over the default binding. As shown in Figure 4, the default binding for the \$project 1 volume is "codasrc". However, as shown in Figure 2, the system configuration file redefines this parameter to "synrgensrc" for synrgenhackers.

Arbitrary C code (surrounded by ' $\{$ ' and ' $\}$ ') also appears in many places within the body of Figure 4 . By allowing experimenters to combine specialized syntactic constructs together with arbitrary $\mathrm{C}$ code, SynRGen provides a good balance between brevity and open-endedness.

\subsection{Implementation Status}

SynRGen has been operational since May 1992. The implementation is highly portable and has run under Mach on DEC MIPS workstations, Intel 386/486 machines, and IBM RTs. It has also been ported to run under AIX on IBM RS/6000 machines. The three preprocessors are implemented in $\mathrm{C}$, using lex and yacc.

We have built up a small library of parameterized micromodels representing a variety of typical applications used in our environment. These include use of an emacs-style editor, $\mathrm{C}$ compilation, and building programs via make. Using micromodels and user classes representative of our environment, we have used SynRGen extensively for stress testing new releases of the Coda File System. Also, we have conducted controlled experiments to evaluate how realistically SynRGen models a true Coda file server workload. The results of our experiments are reported in the next section. 


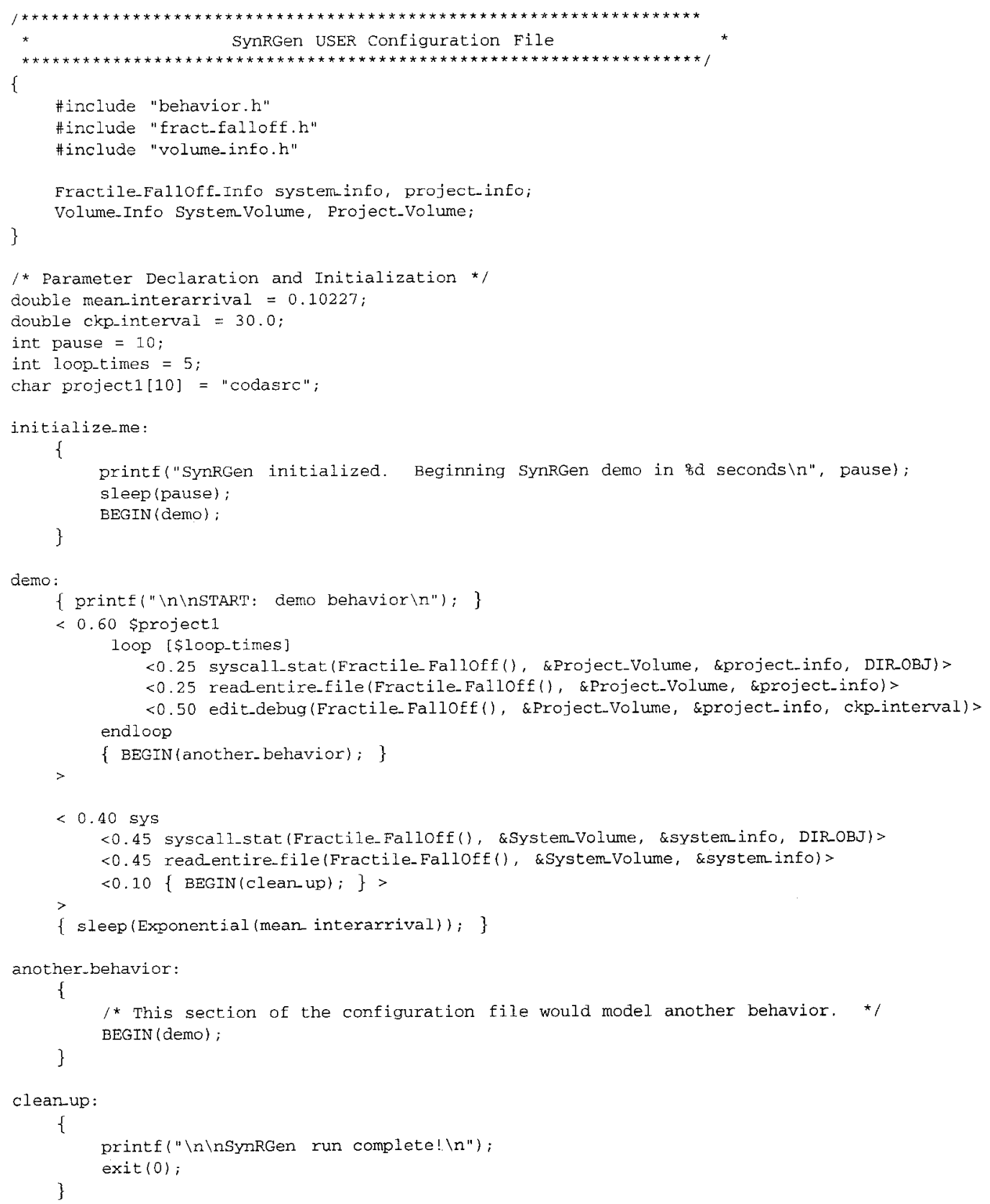

This figure shows the user class configuration file corresponding to the user class finite state machine shown in Figures 1 (a) and (b). The demo behavior exemplifies SynRGen's syntactic constructs which simplify modeling common control flows. In this behavior, we see three distinct actions syscall_stat, read_entire_file, and edit_debug. These actions correspond to micromodels. Arbitrary $\mathrm{C}$ code appears in numerous places in the file: at the top of the file, in well-defined points within the demo behavior, and as the entirety of three behaviors (initialize_me, another_behavior, and clean_up). The BEGIN statements that appear throughout this file represent transitions between behaviors. Although the Fractile_falloff() parameter looks like a function call, the preprocessor translates this into a pointer to a function so that the micromodels can use it as a pathname iterator.

Figure 4: Sample User Class Configuration File 


\section{Case Study: Users in an Edit/Debug Cycle}

An important question is "How well does a SynRGen workload emulate a real workload?" To answer this question, we conducted a performance-oriented study [6] comparing real users to a synthetic user. We chose to study the edit/debug cycle because it is a common activity in our environment as well as many others. We wrote micromodels for the most frequent activities in an edit/debug cycle, generated a synthetic user, and then performed a controlled experiment to compare the load generated on Coda servers by the synthetic user to that generated by real users.

\subsection{The Synthetic User}

Our first task was to build micromodels of the tools most frequently used during edit/debug cycles. To build these micromodels we examined many traces generated by a number of users working in an edit/debug cycle on a variety of machine architectures.

After completıng the micromodels, we constructed a synthetic user. The main activity of this synthetic user is an edit/debug cycle that consists of editing some number of files associated with a particular "program", recomplling the modified files, and executing the program after it is built In addition, the synthetic user occasionally performs a few other related activities such as consulting a manual page, examining a system header file or looking through a source file. Broadly, the user resembles the example shown in Figure 4. The important user class parameters are the mean interarrival time of file system requests, and the mean checkpoint interval time for the emacs-style editor.

\subsection{The Experiment}

For our experiment, we observed five $\mathrm{C}$ or $\mathrm{C}++$ programmers as they performed edit/debug activity for one hour. We made no attempt to rigidly constrain these users - our only request was that they confine their activities to a single workstation. Inevitably, some activities not modeled by SynRGen were performed, but we believe that the amount of such activity was minimal

These users were working on DEC DS-5000/200 machines running Mach 2.6. The data they were accessing resided primarily in triply replicated volumes located on three Coda servers. Each server was also a DS-5000/200 machine running Mach 26 The client and server machines communicated via an Ethernet.

The system parameters we measured on the Coda servers include distribution of incoming RPC operations. transactional activity, CPU utilization, disk activity, and Ethernet load. In addition to these server and network statistics, we obtained file reference traces of these users on the client machines.
The mean interarrival time parameter of our synthetic user was set to the mean interarrival time of file system requests observed in the traces of these users (about 0.1 seconds). The mean checkpoint interval parameter to our synthetic user was set using an estimate of the frequency of checkpoints in actual use of our editors (30 seconds) In addition, the pathname iterator to our micromodels was the fractile falloff distribution described by Satyanarayanan [15]. Specifically, $75 \%$ of the time, $4 \%$ of the files were referenced; $20 \%$ of the time, $16 \%$ of the files were referenced; and the remaining $5 \%$ of the time, the other $80 \%$ of the files were referenced.

Using these parameters, we then ran the synthetic user on one of the client workstations in a subtree of the Coda file system. The physical characteristics of files in this subtree were consistent with the data presented in Table 1 for project volumes.

\subsection{Results}

Table 2 compares the values of the system variables obtained from our real users to those obtained from our synthetic user. The last column of this table calculates the difference between the synthetic user and the mean of the real users in units of the standard deviation of the real users. This column shows that all but two system variables (the number of status fetches and the CPU usage in user mode) fall within one standard deviation of the average for the real users. For most system variables including the two above, the synthetic user comes within $20 \%$ of the mean value for the real users. With the exception of the number of data fetch and store requests. all the other system variables of interest lie within about $40 \%$ of their observed values for the real users In all cases where the results from the synthetic user diverge substantially from the mean value for the real users, the result from the synthetic user still falls within the range observed for real users.

A final observation is that SynRGen consistently underestimates the observed values from real users (i.e., relative error is negative for most system variables). This suggests that we might be able to get the synthetic user to better match real users by applying a correction factor to the run time parameters. Experiments not reported here confirm that this is indeed the case.

It is important to note that the bulk of our effort in this experiment was in building the micromodels The actual construction of our synthetic user was relatively simple. This confirms the underlying hypothesis of our approach: that it is possible to substantially separate the efforts of the modeler and experimenter, and to encapsulate the work of the former in micromodels

Our results confirm that SynRGen is able to realistically model users in at least one domain. Further validation of our approach would require simılar controlled experiments spanning a broader class of activitıes, applications, and environments. 


\begin{tabular}{|c|c|c|c|c|c|c|c|c|c|c|}
\hline \multirow[b]{2}{*}{ Characteristic } & \multicolumn{7}{|c|}{ Real Users } & \multirow{2}{*}{$\begin{array}{c}\text { Synthetic } \\
\text { User }\end{array}$} & \multirow{2}{*}{$\begin{array}{c}\text { Relative } \\
\text { Error }\end{array}$} & \multirow{2}{*}{$\begin{array}{l}\text { Stdzd } \\
\text { Error }\end{array}$} \\
\hline & User 1 & User 2 & User 3 & User 4 & User 5 & Mean (StDev) & $\mathrm{COV}$ & & & \\
\hline Total Time (sec) & 3642 & 3379 & $36 \overline{03}$ & 3602 & 3597 & $3565(105)$ & 0.0 & 3602 & $1 \%$ & 0.4 \\
\hline Total Operations & 3431 & 2177 & 2898 & 2085 & 2601 & $2638(551)$ & 0.2 & 2590 & $-2 \%$ & 0.1 \\
\hline SERVEROPB: & & & & & & & & & & \\
\hline FetchData & 32 & 30 & 63 & 4 & 28 & $31(21)$ & 0.7 & 11 & $-65 \%$ & 1.0 \\
\hline FetchStatus & 1219 & 1061 & 1462 & 1161 & 1278 & $1236(149)$ & 0.1 & 1078 & $-13 \%$ & 1.1 \\
\hline StoreData & 269 & 29 & 70 & 72 & 35 & $95(99)$ & 1.0 & 167 & $76 \%$ & 0.7 \\
\hline StoreStatus & 263 & 48 & 49 & 59 & 21 & $88(99)$ & 1.1 & 115 & $31 \%$ & 0.3 \\
\hline Create & 117 & 26 & 21 & 22 & 7 & $39(44)$ & 1.2 & 33 & $-15 \%$ & 0.1 \\
\hline RVM: & & & & & & & & & & \\
\hline Transactions & 2158 & 437 & 422 & 403 & 394 & $763(780)$ & 1.0 & 686 & $-10 \%$ & 0.1 \\
\hline CPU TIME: & & & & & & & & & & \\
\hline User (sec) & 81 & 90 & 81 & 63 & 68 & $77(11)$ & 0.1 & 63 & $-18 \%$ & 1.3 \\
\hline System (sec) & 163 & 169 & 244 & 132 & 133 & $168(46)$ & 0.3 & 134 & $-20 \%$ & 0.7 \\
\hline DISK: & & & & & & & & & & \\
\hline Transfers & 7804 & 2434 & 11735 & 2535 & 3518 & $5605(4070)$ & 0.7 & 3968 & $-29 \%$ & 0.4 \\
\hline KBs Transferred & 57112 & 15891 & 86862 & 15046 & 22359 & 39454 (31633) & 0.8 & 22420 & $-43 \%$ & 0.5 \\
\hline Busy Time (sec) & 137 & 45 & 196 & 46 & 65 & $98(67)$ & 0.7 & 69 & $-30 \%$ & 0.4 \\
\hline ETHERNET: & & & & & & & & & & \\
\hline Packets In & 123800 & 129365 & 163207 & 117353 & 107830 & $128311(21088)$ & 0.2 & 114245 & $-11 \%$ & 0.7 \\
\hline Packets Out & 27485 & 34737 & 38847 & 25867 & 24888 & 30365 (61 15) & 0.2 & 25205 & $-17 \%$ & 0.8 \\
\hline
\end{tabular}

This table presents the results of a controlled experiment comparing the workload generated on Coda servers by five real users to that generated by a synthetuc user For each "user", we present the mean value observed at the three servers. In addition to the load generated by each real user, we present the mean, standard deviation and coefficient of variation for these users The relative error is defined as the ratio of the difference between the synthetic user and the mean of the real users to the mean of the real users. A relative error greater than zero implies that the synthetic load overestimated the actual load. while an error less than zero implies the synthetic load underestımated The standardized error is defined as the ratio of the absolute value of the difference between the synthetic user and the mean of the real users to the standard deviation of the real users.

Table 2: Comparison of Real and Synthetic Users

\subsection{Extending the Case Study}

In the above sections we used SynRGen to model a single user in an edit-debug cycle. Suppose, however, that we need to model an entire user community. What would the necessary changes be? The first step is creating validated SynRGen models for each class of user in the community under study. Once available, these synthetic users can be run simultaneously to generate a workload representative of the entire community.

Almost certainly, we will want to model data sharing between users. Sharing can be read-read, read-write or write-write. For simplicity, we refer to read-read sharing simply as "read-sharing" and to both read-write and write-write sharing as "write-sharing".

We model read- and write-sharing of files and read-sharing of directories by having synthetic users perform activities in shared volumes. For example, to model read-sharing of manual pages or header files, all members of a community might occasionally examine the contents of files and directories in shared system volumes. Similarly, when modeling members of a project, one would concentrate their activities in one or more project volumes. Increasing the time spent performing activities in the shared volume will increase the probability of sharing.

Modeling write-sharing of directories is more challenging. Synthetic users create, remove and rename objects as dictated by their micromodels When synthetic users write-share directories, they may experience interference caused by these directory updates. For instance, one synthetic user might remove an object that another synthetic user later attempts to edit. The micromodels in Section 4.1 do not update internal data structures upon discovering new, missing or renamed objects. In order to support write-sharing of directories, we must modify those micromodels to use failed system calls as hints to trigger updates to internal data structures. One of SynRGen's strengths is that these improvements require modifications only to the micromodels and not to SynRGen itself.

\section{Open Issues and Future Work}

\subsection{Automatic Micromodeling}

Because writing micromodels is a labor intensive task, automatıng this process in some fashion would make modeling new activities easier. One approach would be to "invert" a file reference trace, producing a command script. This command script, when executed, would produce a trace isomorphic to the original trace We have experience with an untrace facility that does just that [17]. A difficulty with such an approach is determining how to parameterize the generated micromodels, and how to specify locality.

Another approach would be to use the strategy proposed by 
Thekkath et al. [20]. This approach recognizes that random samples selected from a representative trace of the workload being modeled will result in workloads that exhibit the same statistical characteristics as the original. These random samples could be used as micromodels. Parameterizing these micromodels must still be done manually

Both of these techniques require an accurate trace of the environment being modeled This requirement limits their applicability to environments that already exist - one cannot use either of these techniques for constructung a micromodel for a hypothetical or anticipated application.

\subsection{Incorporating Locality Phases}

SynRGen provides a means of modeling locality of reference in a synthetic workload In Bunt et al.'s terminology [2, 3]. SynRGen offers flexibility in modeling any degree of concentration of locality; that is, SynRGen allows any number of files to be in the active set. However, Majumdar and Bunt have shown that file reference locality exhibits phases, or bounded localty intervals [8] Although we do not foresee any difficulty in incorporating locality phases, SynRGen currently has no notion of these phases The consequence of not supporting bounded locality intervals is that SynRGen can be expected to display a higher degree of locality than actual workloads Adding the notion of locality phases to SynRGen would improve the accuracy of modeling.

As seen in Figure 2, the synthetic user greatly underestimated the number of fetches seen by the servers One possible explanation of this behavior is that the client cache was able to service fetch requests for the synthetic user more successfully than for the real users. In other words, the synthetic user may be displaying a higher degree of locality than real users.

\subsection{Quantifying Accuracy of Micromodels}

The overall realism of an experiment is limited by the accuracy of individual micromodels. This raises the question of how to quantify the accuracy of a micromodel. One approach would be to define some metrics and use these metrics to compare traces generated by the synthetic user to a set of reference traces generated in a real system. These metrics might include the fraction of files referenced and the locality of files referenced. While such metrics give some indication of the accuracy of the micromodel, they completely ignore the order in which events happen. In modeling locality of reference, it is important to capture the order in which system calls occur as well as the order in which files and directories are accessed.

One metric that respects ordering is the longest common subsequence (LCS) The LCS is a well-known measure of closeness between two strings and has been used in a variety of contexts such as DNA sequencing and speech processing[13] We explored the possibility of using this metric to quantify the accuracy of micromodels. Unfortunately, we found this approach to be intractable due to the large storage requirements of the algorithm and the voluminous size of realistic traces.

Quantifying accuracy thus remains an open problem. We now believe that such quantification must be based either on metrics other than the LCS, or on a more efficient approximation to the LCS.

\section{Related work}

Synthetic file reference generation has received considerable attention in the recent past Much of this attention has been focused on measuring NFS performance. For example, NHFSStone [9] facilitates comparisons of competing NFS implementations. A fundamental distinction between this work and ours is the dependence of this benchmark on the NFS interface. Further, this benchmark is considerably less realistic and less flexible than SynRGen.

Bodnarchuk and Bunt [1] significantly improve upon the above benchmark in both flexibility and realism. Their algorithm involves sampling discrete distributions to choose an NFS operation. a file system in which to operate (uniformly choosing a specific file from a "representative set" of this file system), a data transfer size, and an interarrival time. SynRGen differs from this work in that it raises the generator up to the file system call level, models interfile locality in addition to intrafile locality, and allows measurement of client effectiveness as well as server and network effectiveness.

In contrast to NFS benchmarks. SynRGen operates at the file system call level. This makes performance comparison of file system implementations such as AFS and NFS possible. It also broadens the range of phenomena that can be modeled

Another widely used benchmark, the Andrew Benchmark[7], operates at the Unix system call level and attains a respectable degree of realism. Because this benchmark is restricted to specific activities, it cannot be used to model a variety of workloads. In contrast, SynRGen is considerably more flexible and allows a wide range of scenarios to be modeled.

Viewing SynRGen in a broader context, it is clear that the idea of generating a synthetic workload is not new. In fact, there are a wide variety of synthetic workload generators including the SPEC benchmark suite[19], the TPC benchmarks[18], IOBENCH[21], tcplib[5], and many others. What differentiates SynRGen from other workload generators is its flexibility in accommodating new workloads while preserving realism. It achieves this fexibility by providing not a single workload generator, but a common framework on which workload generators can be built. The separation of micromodels from their stochastic combination is, to the best of our knowledge, not duplicated in any other synthetic workload generator. 


\section{Conclusion}

SynRGen is a tool born of necessity. Early in the evolution of the Coda File System, it became clear to us that we needed some way of stressing our system without burdening real users. In retrospect, SynRGen has indeed proven to be an invaluable tool for this purpose. Coda is now used daily by over 30 users as their primary data repository. New releases of Coda are exposed to synthetic users for an extended period of time before they are installed on our production servers. Furthermore, SynRGen proved invaluable in debugging our current backup system.

We are confident that SynRGen's unique ability to combine realism with flexibility will make it attractive to other file system developers. As discussed earlier in the paper, we foresee it being useful in performance evaluation. We also envision it allowing researchers to subject their systems to a broader range of workloads than has historically been possible. This will enhance the credibility of research systems, whose generality has often been questioned because of their bias toward academic workloads.

While already useful in its present form, SynRGen is not a finished piece of work. There is clearly work to be done in building up a rich library of micromodels, representing a wide range of applications. A related piece of work is to assemble a collection of configuration files capable of representing a variety of usage environments. Finally, as Section 5 explained, SynRGen itself can be refined in a number of ways. We believe that these efforts will result in an important asset to the file systems community.

\section{Acknowledgements}

We wish to thank Lily Mummert for her indispensable file reference tracing facility. In addition, we would also like to thank our "real" users and those who provided comments on early versions of this paper, including Puneet Kumar, Qi Lu, Scott Nettles, Brian Noble, Chris Okasaki, Josh Raiff. David Steere and Matt Zekauskas. Finally, we would like to thank the referees for their many valuable comments.

\section{References}

[1] Bodnarchuk, R R. AND BUNT, R B A Synthetic Workload Model for a Distributed System File Server. In Proceedings of the 1991 ACM SIGMETRICS Conference on Measurement and Modeling of Computer Systems (San Diego. CA, May 1991).

12] BUNT, R. B., AND MURPHY, J M The Measurement of Locality and the Behavior of Programs The Computer Journal 27, 3 (August 1984)

[3] BUNT, K У, MURPHY J M ANDMaJUMDaR S A Mitdure or PIOgram Locallity and Its Application In Proceeding of the 1984 ACM SIGMETRICS Conference on Measurement and Modeling of Computer Systems (Cambridge, MA, August 1984)
[4] CORMEN, T H, LEISERSON, C E , AND RIVEST, R L Introduction to Algorthm: MIT Press, Cambridge, MA, 1990.

[5] Danzig, P. B., Jamin, S., CÁCeres, R., Mitzel. D. J., and Estrin, D An Empirical Workload Model for Driving Wide-area TCP/1P Network Simulations Internetworking: Research and Expertence 3, 1 (March 1992)

[6] FerRaRI, D On the Foundation of Artifical Workload Design In Proceedings of the 1984 ACM SIGMETRICS Conference on Measurement and Modeling of Computer System. (Cambridge, MA. August 1984).

[7] Howard, J H, Kazar, J L, Menees, S G, Nichols, D A, SatyaNARAYANAN, M, AND Sidebotham, $R \mathrm{~N}$ Scale and Performance in a Distributed File System. ACM Transactoons on Computer Systems 6, 1 (February 1988)

[8] MaJUmDar, S., AND BUNT, R. B. Measurement and Analysis of Locality Phases in File Referencing Behavior In Proceeding of Performance 86 and ACM Sigmetrics 86, Joint Conference on Computer Performance Modelling, Measurement and Evaluation (Raleigh, NC. May 1986)

[9] MoLLoY, M. K Anatomy of the NHFSSTONES Benchmarks ACM SIGMETRICS Performance Evaluation Revtew 19, 4 (May 1992)

[10] Mummert, L B, and Satyanarayanan, M Efficient and Portable File Reference Tracing in a Distributed Workstation Environment Carnegie Mellon University, manuscript in preparation.

(11] Nelson, M., Welch, B., AND OUsterhout, J. Caching in the Sprite Network File System ACM Transactoons on Computer Systems 6. 1 (February 1988)

[12] Sandberg, R, Goldberg, D, Kleiman, S, Walsh, D, and Lyon, B Design and Implementation of the Sun Network Filesystem In Proceedings of the USENIX Summer Conference (Summer 1985)

[13] Sankoff, D. And Kruskal, J B Time Warps, Strings Edits, and Macromoleculer The Theory and Practice of Sequence Compartson Addison-Wesley Publishing Company, Inc , Reading, MA, 1983

[14] Satyanarayanan, M The ITC Distributed File System Principles and Design In Proceedings Tenth ACM Symposum on Operating Systems Principles (December 1985)

[15] SatYanarayanan, M. Modelling Storage Syitems. UMI Research Press, Ann Arbor. MI, 1986.

(16) Satyanarayanan. M , Kistler, J J , Kumar, P., Okasaki, M. E, Siegel, E H, AND STEERE, D C Coda A Highly Avalable File System for a Disinbuted Workstatuon Environment IEEE Transactons on Computers 39, 4 (April 1990)

[17] Satyanarayanan, M , Kistler, J 3 , Mummert, L B , Ebling, M R , Kumar. P., AND LU, Q Experience with Disconnected Operation in a Mobile Computing Environment In Proceedings of the 1993 USENIX Sympoxium on Mobile and Locaton-IndependentComputing (Cambridge, MA, August 1993)

[18] SERLIN, O The History of DebitCredit and the TPC In The Benchmark Handbook, J Gray, Ed Morgan Kaufman, 1991.

[19] System Performance Evaluation CoOperative. SPEC Benchmark Sutte Release I O. October 1989

[20] THEKKaTH, C A , WILkES, J , AND Lazowska, E D Techniques for File Syatem Simulation. Technical Report 92-09-08, Unversity of Washington, Department of Computer Science and Engineering, 1992

[21] WOlman, B L, ANd OlSON, T M IOBENCH A System Independent 10 Benchmark Computer Archttecture News 17,5 (September 1989) 